\title{
Administration of a Divided Dose of Carboplatin Following Carboplatin-Induced Syndrome of Inappropriate Secretion of Antidiuretic Hormone (SIADH): A Case Report
}

\author{
Suguru Fujitsuka, Naoki Horikawa*, Teruki Yoshida, Sae Yu, Ryosuke Kuroda, Mitsuru \\ Tsuji, Maki Umemiya, Rei Gou, Yoshihide Inayama, Hirohiko Tani and Kenzo Kosaka
}

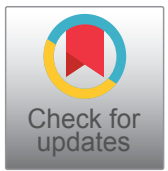

Department of Obstetrics and Gynecology, Shizuoka General Hospital, Shizuoka, Japan

*Corresponding author: Dr. Naoki Horikawa, MD, PhD, Department of Obstetrics and Gynecology, Shizuoka General Hospital, 4-27-1, Kita-ando, Aoi-ku, Shizuoka City, Shizuoka 420-8527, Japan, Tel: +8154-247-6111, Fax: +8154-247-6111

\begin{abstract}
Chemotherapy-induced severe hyponatremia is a lifethreatening condition. Platinum-based agents play a key role in ovarian cancer treatment, but they are more likely to cause hyponatremia than other anti-cancer agents. The optimal strategy for treating ovarian cancer in the case of severe hyponatremia induced by the platinum agent remains unclear. We report the case of 77 -year-old women with multiple peritoneal recurrences five years and eight months after surgery for ovarian clear cell carcinoma. She received tri-weekly docetaxel and carboplatin (DC) therapy and bevacizumab, following which she developed severe hyponatremia due to syndrome of inappropriate antidiuretic hormone secretion (SIADH). We were reluctant to change the drugs because they effectively reduced the cancer antigen 125 level. The chemotherapy regimen was changed to weekly administration off a divided dose of DC, and she completed six cycles of weekly DC without electrolyte disturbance, including hyponatremia, or tumor recurrence. Weekly carboplatin administration may be a promising alternative to tri-weekly carboplatin administration after the development of carboplatin-induced SIADH.
\end{abstract}

\section{Keywords}

Hyponatremia, Syndrome of inappropriate antidiuretic hormone secretion, Carboplatin, Divided dose

\section{Abbreviations}

AUC: Area Under the Curve; CA125: Cancer Antigen 125; SIADH: Syndrome of Inappropriate Antidiuretic Hormone Secretion; RSWS: Renal Salt Wasting Syndrome

\section{Introduction}

Hyponatremia occurs in $2.6 \%-29.1 \%$ of patients receiving chemotherapy [1]. Although platinum-based agents play a key role in ovarian cancer treatment, they are more likely to cause hyponatremia than nonplatinum-based agents (incidence of hyponatremia: $11.9 \%$ and $3.8 \%$, respectively) [1]. In previous reports, platinum-based drugs were often replaced with nonplatinum-based drugs or other platinum-based drugs or discontinued due to severe drug-induced hyponatremia; however, the optimal strategy is still unknown. Here, we report a case of severe hyponatremia following tri-weekly carboplatin administration for treating recurrent ovarian clear cell carcinoma. We changed the regimen to weekly administration by dividing the dose of carboplatin, and no hyponatremia occurred despite not changing the drugs.

\section{Case Description}

A 77-year-old woman underwent total abdominal hysterectomy, bilateral salpingo-oophorectomy, partial omentectomy, and pelvic and para-aortic lymphadenectomy for treating right ovarian cancer. The postoperative diagnosis was right ovarian clear cell carcinoma (stage IC3). She received adjuvant chemotherapy with paclitaxel $\left(75 \mathrm{mg} / \mathrm{m}^{2}\right)$ and carboplatin (area under the curve [AUC]: 2) on days 1 , 8 , and 15 . On day eight, the regimen was discontinued

Citation: Fujitsuka S, Horikawa N, Yoshida T, Yu S, Kuroda R, Tsuji M, et al. (2021) Administration of a Divided Dose of Carboplatin Following Carboplatin-Induced Syndrome of Inappropriate Secretion of Antidiuretic Hormone (SIADH): A Case Report. Obstet Gynecol Cases Rev 8:207. doi. org/10.23937/2377-9004/1410207

Accepted: July 28, 2021: Published: July 30, 2021

Copyright: @ 2021 Fujitsuka S, et al. This is an open-access article distributed under the terms of the Creative Commons Attribution License, which permits unrestricted use, distribution, and reproduction in any medium, provided the original author and source are credited. 
because of elevated serum liver enzyme levels due to drug-induced liver injury (aspartate aminotransferase: $284 \mathrm{U} / \mathrm{L}$, alanine aminotransferase: $344 \mathrm{U} / \mathrm{L}$, and lactate dehydrogenase: $406 \mathrm{U} / \mathrm{L}$ ). The regimen was modified to irinotecan $\left(60 \mathrm{mg} / \mathrm{m}^{2}\right.$ on days 1,8 , and 15$)$ and cisplatin ( $60 \mathrm{mg} / \mathrm{m}^{2}$ on day one). However, on day six, she experienced convulsions and hyponatremia (serum sodium level: $109 \mathrm{mEq} / \mathrm{L}$ ). She was treated with supplemental sodium chloride, and her sodium level recovered six days after the onset of convulsions. Hence, the patient was followed without adjuvant chemotherapy. Five years and eight months after the surgery, the patient's cancer antigen 125 (CA125) level was elevated to $569 \mathrm{U} / \mathrm{L}$, and positron emission tomography/computed tomography showed multiple peritoneal dissemination, indicating recurrence of ovarian cancer (Figure 1). She received chemotherapy with docetaxel ( $70 \mathrm{mg} / \mathrm{m}^{2}$ on day one), carboplatin (AUC: 6 on day one), and bevacizumab ( $15 \mathrm{mg} / \mathrm{kg}$ on day one) because of her history of liver injury and hyponatremia following paclitaxel and cisplatin administration, respectively. On day five, the patient complained of general malaise. Hematological examination revealed a serum sodium level of $115 \mathrm{mEq} / \mathrm{L}$, antidiuretic hormone level of $7.5 \mathrm{pg} / \mathrm{mL}$, creatinine level of $0.52 \mathrm{mg} / \mathrm{dL}$, and cortisol level of $31.5 \mu \mathrm{g} / \mathrm{dL}$, with a plasma osmolality of $236 \mathrm{mOsm} / \mathrm{kg}$, and urine osmolality of $631 \mathrm{mOsm} /$ $\mathrm{kg}$. Physical examination did not reveal any symptoms of hypovolemia or hypervolemia. She was diagnosed with carboplatin-induced syndrome of inappropriate
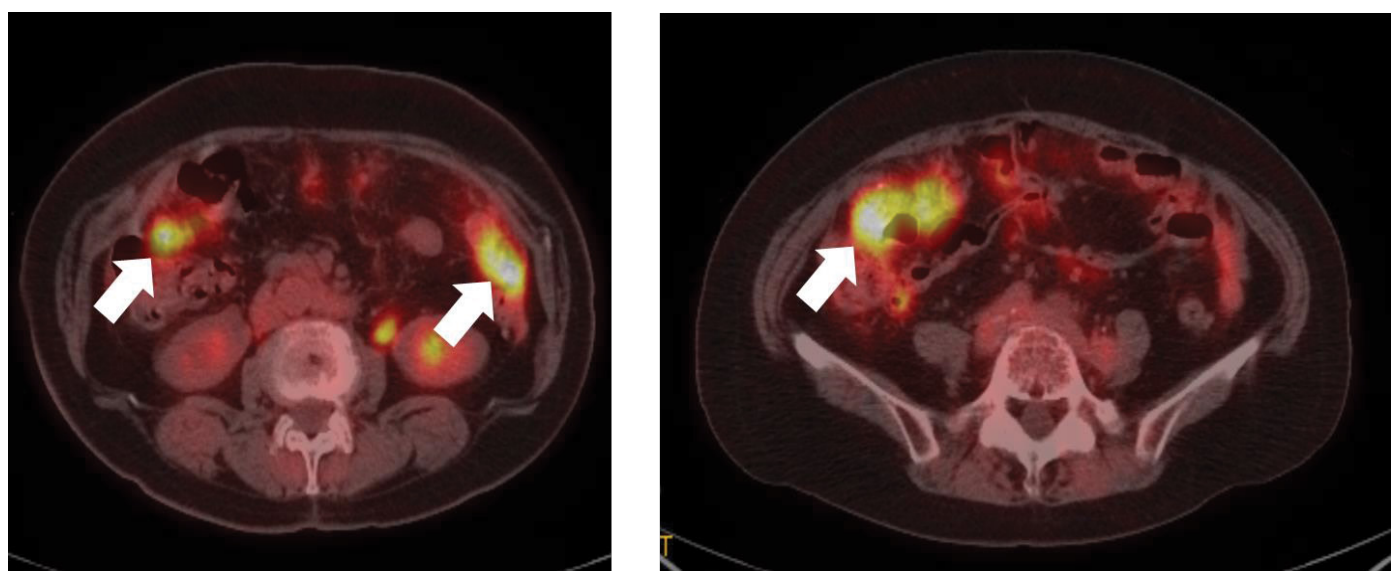

Figure 1: Positron emission tomography/computed tomography image showing multiple peritoneal dissemination of recurrent ovarian cancer (white arrows).

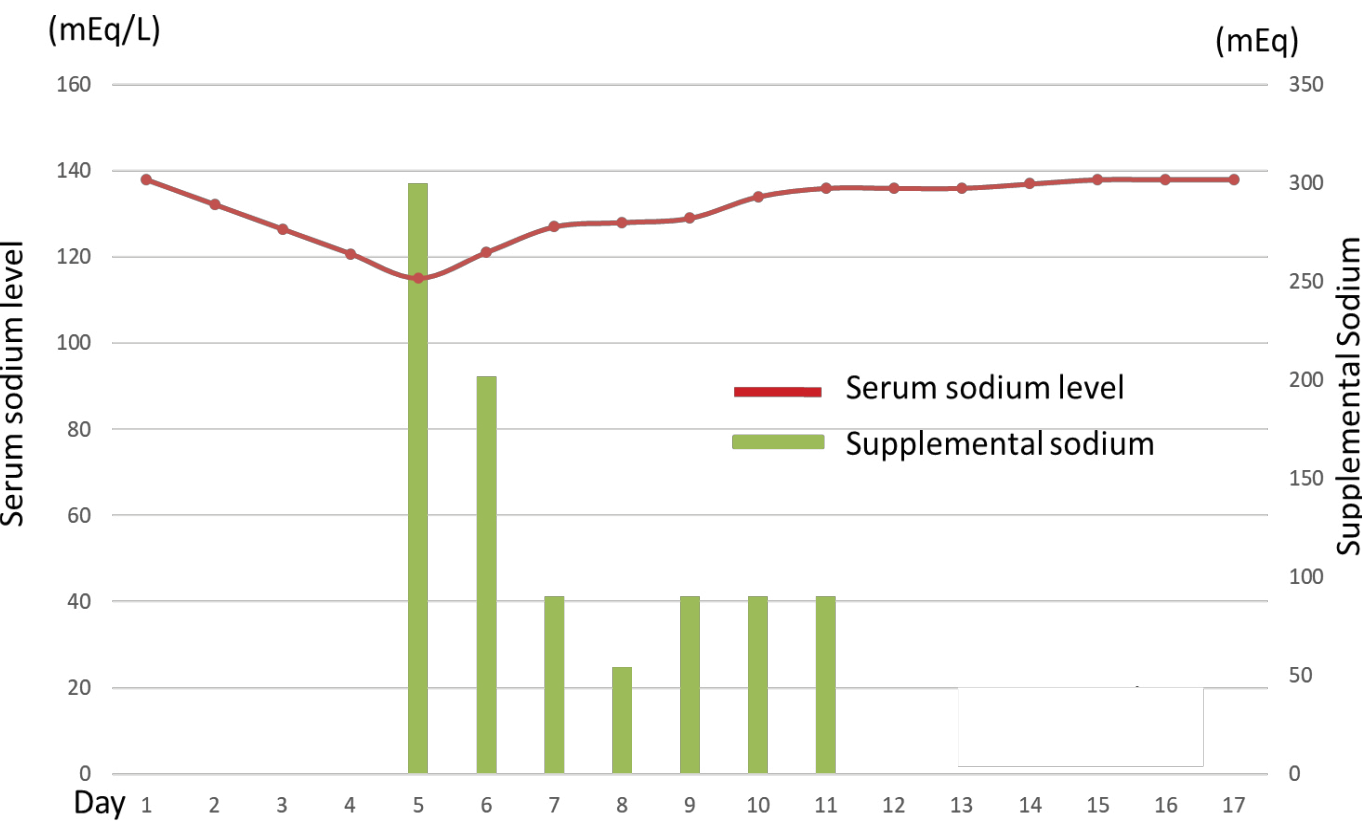

Figure 2: The patient's serum sodium level decreased to $115 \mathrm{mEq} / \mathrm{mL}$ on day five of chemotherapy. It was corrected with supplemental sodium, which was discontinued on day 21. Inappropriate ADH secretion occurred on day 5 of chemotherapy, but its serum level was within the normal range on day 15.

ADH: Antidiuretic hormone. 
antidiuretic hormone secretion (SIADH) and treated with hypertonic saline infusion. Her serum sodium level gradually rose to normal levels eight days after sodium supplementation (Figure 2). Although SIADH occurred, the CA125 level reduced to $66 \mathrm{U} / \mathrm{L}$, demonstrating the efficacy of the regimen. The patient received chemotherapy with divided doses of docetaxel $(35 \mathrm{mg} /$ $\mathrm{m}^{2}$ on days 1,8 , and 15 ) and carboplatin (AUC: 2 on days $1,8,15)$, for which she provided informed consent. After weekly administration of docetaxel and carboplatin (weekly DC), her CA125 levels further decreased to normal levels. She completed six cycles of weekly DC without electrolyte disturbance or tumor recurrence.

\section{Conclusions}

Platinum-based agents have been known to cause SIADH and renal salt wasting syndrome (RSWS), leading to hyponatremia. SIADH is a disorder of impaired water excretion due to the over-secretion of $\mathrm{ADH}$. Its treatment includes fluid restriction and sodium supplementation. The occurrence of SIADH has been associated with ectopic ADH-producing tumors, lung disease, surgical stress, central nervous system disease, and the use of certain medication, as was the case here [2]. In RSWS, impaired proximal tubular reabsorption of sodium and water induces hyponatremia and dehydration; it is often caused by platinum-based agents. The treatment of RSWS includes fluid replacement. Differential diagnosis is difficult but important because the treatment strategy differs [3].

Ezoe, et al. reported that cisplatin caused hyponatremia more often than carboplatin; the incidence of grade $3 / 4$ hyponatremia was $13.5 \%$ in patients treated with cisplatin and $7.6 \%$ in those treated with carboplatin [1]. They attributed this difference to nephrotoxicity due to the mechanism of cisplatin excretion [4]. We have listed the previous reports of carboplatin-induced hyponatremia in Table 1. Only five cases of hyponatremia after carboplatin administration have been reported; among them, one patient had RSWS [5] and the other four had SIADH [6-9]. While RSWS is a common cause of cisplatin-induced hyponatremia [10], SIADH is the most common cause of carboplatin-induced hyponatremia.

Our patient did not have dehydration or renal dys- function, which indicated that SIADH was the most likely cause of the hyponatremia. Grade 4 hyponatremia occurred after the administration of docetaxel, carboplatin, and bevacizumab. There are no reports of bevacizumab-induced hyponatremia, and only one case of nab-paclitaxel-induced hyponatremia has been reported [11]. Our patient's SIADH might have been caused by the administration of carboplatin (AUC: 6). In previous reports, carboplatin administration was continued in only one of four patients who developed carboplatin-associated SIADH [9], and the drug was changed or discontinued in the other three [6-8]. The patient in whom carboplatin administration was continued had grade 3 hyponatremia, which recovered following oral sodium supplementation, and chemotherapy was discontinued after two cycles because the regimen was not effective [9].

In this case, we administered a divided dose of DC, allowing us to continue chemotherapy without reducing the total dose of carboplatin, which was found to be effective, and without the patient developing SIADH. Recurrent ovarian clear cell carcinoma is resistant to chemotherapy [12]; therefore, it is important to avoid discontinuing the use of platinum-based agents. The MITO-7 [13] and ICON-8 [14] trials found that a weekly regimen of carboplatin and paclitaxel was not significantly inferior to a tri-weekly regimen for the treatment of ovarian cancer. Although bevacizumab has been shown to be beneficial for the treatment of recurrent ovarian clear cell carcinoma [15], we did not administer bevacizumab to our patient because a regimen of bevacizumab combined with weekly DC therapy is not adopted at our hospital.

Weekly carboplatin administration may be a promising alternative to tri-weekly carboplatin administration after the development of carboplatin-induced SIADH.

\section{Acknowledgments}

The authors declare no conflicts of interest. We would like to thank Editage (https://www.editage.com/) for English language editing.

\section{Sources of Support}

All authors contributed equally to this work.

Table 1: Reports of carboplatin-induced syndrome of inappropriate of antidiuretic hormone secretion.

\begin{tabular}{|l|l|l|l|l|l|}
\hline Reporter & Age/Sex & Tumor type & Chemotherapy regimen & $\begin{array}{l}\text { Nadir serum } \\
\text { sodium level } \\
\text { (m Eq/L) }\end{array}$ & Next treatement for tumor \\
\hline Yokoyama & $63 / F$ & Ovarian cancer & CBDCA + PTX & 109 & CDDP + PTX \\
\hline Fujioka & $60 / F$ & Lung cancer & CBDCA + PTX & 101 & No record \\
\hline Turner & $49 / F$ & Breast cancer & CBDCA + DTX + HER & 105 & DTX + HER (without CBDCA) \\
\hline Sugiyama & $66 / F$ & Urethral cancer & CBDCA + PTX & No record & Continue (SIADH was controllable) \\
\hline Our case & 77/F & Ovarian cancer & CBDCA + DTX + BEV & 115 & CBDCA + DTX (weekly regimen) \\
\hline
\end{tabular}

CBDCA: Carboplatin; PTX: Paclitaxel; CDDP: Cisplatin; DTX: Docetaxel; HER: Trastuzumab; SIADH: Syndrome of inappropriate antidiuretic hormone secretion; BEV: Bevacizumab; BSC: Best supportive care. 


\section{References}

1. Ezoe $\mathrm{Y}$, Mizusawa J, Katayama H, Kataoka K, Muto M (2016) An integrated analysis of hyponatremia in cancer patients receiving platinum-based or nonlatinum-based chemotherapy in clinical trials (JCOG1405-A). Oncotarget 9: 6596-6606.

2. Cuesta M, Thompson CJ (2016) The syndrome of inappropriate antidiuresis (SIAD). Best Pract Res Clin Endocrinol Metab 30: 175-187.

3. Yeoh KW, Camilleri P, Patel K (2010) Chemotherapy induced hyponatraemia. BMJ Case Rep.

4. Siddik ZH, Newell DR, Boxall FE, Harrap KR (1987) The comparative pharmacokinetics of carboplatin and cisplatin in mice and rats. Biochem Pharmacol 36: 1925-1932.

5. Tschering $\mathrm{C}$, Rubie H, Chancholle A, Claeyssens V, Robert A, et al. (2003) Recurrent renal salt wasting in a child treated with carboplatin and etoposide. Cancer 73: 1761-1763.

6. Yokoyama Y, Shigeto T, Futagami M, Mizunuma H (2005) Sydrome of inappropriate secretion of anti-diuretic hormone following carboplatin-aclitaxel administration in a patient with recurrent ovarian cancer. Eur J Gynaecol Oncol 26: 531-532.

7. Fujioka S, Nakamura H, Miwa K, Taniguchi Y, Haruki $\mathrm{T}$, et al. (2011) Syndrome of inappropriate secretion of antidiuretic hormone (SIADH) following carboplatinpaclitaxel administration in a patient with lung cancer. Pharmazie 66: 729-730.

8. Turner N, Stewart J, Barnett F, White S (2012) Syndrome of inappropriate anti diuretic hormone secretion secondary to carboplatin after docetaxel-carboplatin- trastuzumab combination for early stage HER-2 positive breast cancer. Asia Pac J Clin Oncol 8: 9-11.
9. Sugiyama $\mathrm{Y}$, Naiki T, Kondo $\mathrm{M}$, lida $\mathrm{K}$, Kondo $\mathrm{Y}$, et al. (2017) Syndrome of inappropriate secretion of antidiuretic hormone caused by carboplatin after switching from cisplatin in a metastatic urethral cancer patient. Urol Case Rep 12: 17-19.

10. lyer AV, Krasnow SH, Dufour DR, Arcenas AS (2003) Sodium-wasting nephropathy caused by cisplatin in a patient with small-cell lung cancer. Clin Lung Cancer 5: 187-189.

11. Neuzillet C, Babai S, Kempf E, Pujol G, Rousseau B, et al. (2016) Severe hyponatremia caused by nab-paclitaxelinduced syndrome of inappropriate antidiuretic hormone secretion: A case report in a patient with metastatic pancreatic adenocarcinoma. Medicine (Baltimore) 95: e4006.

12. Pectasides D, Fountzilas G, Aravantinos G, Kalofonos C, Efstathiou $\mathrm{H}$, et al. (2006) Advanced stage clear-cell epithelial ovarian cancer: The hellenic cooperative oncology group experience. Gynecol Oncol 102: 285-291.

13. Pignata S, Scambia G, Katsaros D, Gallo C, PujadeLauraine E, et al. (2014) Carboplatin plus paclitaxel once a week versus every 3 weeks in patients with advanced ovarian cancer (MITO-7): A randomised, multicentre, openlabel, phase 3 trial. Lancet Oncol 15: 396-405.

14. Clamp AR, James EC, McNeish IA, Dean A, Kim JW, et al. (2019) Weekly dose-dense chemotherapy in first-line epithelial ovarian, fallopian tube, or primary peritoneal carcinoma treatment (ICON8): Primary progression free survival analysis results from a GCIG phase 3 randomised controlled trial. Lancet 394: 2084-2095.

15. Gallego A, Ramon-Patino J, Brenes J, Mendiola M, Berjon A, et al. (2021) Bevacizumab in recurrent ovarian cancer: Could it be particularly effective in patients with clear cell carcinoma? Clin Transl Oncol 23: 536-542. 